\title{
Reduction of CT artifacts from cardiac implantable electronic devices using a combination of virtual monoenergetic images and post-processing algorithms
}

\author{
Lenhard Pennig ${ }^{1}$ (D) David Zopfs ${ }^{1} \cdot$ Roman Gertz $^{1}$ • Johannes Bremm ${ }^{1} \cdot$ Charlotte Zaeske $^{1} \cdot$ Nils Große Hokamp ${ }^{1}$. \\ Erkan Celik $^{1}$ - Lukas Goertz ${ }^{1}$. Marcel Langenbach ${ }^{1}$. Thorsten Persigehl ${ }^{1}$ - Amit Gupta ${ }^{2,3}$ • Jan Borggrefe ${ }^{1,4}$. \\ Simon Lennartz ${ }^{1,5,6} \cdot$ Kai Roman Laukamp ${ }^{1,2,3}$
}

Received: 19 October 2020 / Revised: 16 December 2020 / Accepted: 4 February 2021 / Published online: 25 February 2021

(C) The Author(s) 2021, corrected publication 2021

\begin{abstract}
Objectives To evaluate the reduction of artifacts from cardiac implantable electronic devices (CIEDs) by virtual monoenergetic images (VMI), metal artifact reduction (MAR) algorithms, and their combination $\left(\mathrm{VMI}_{\mathrm{MAR}}\right)$ derived from spectral detector CT (SDCT) of the chest compared to conventional CT images (CI).

Methods In this retrospective study, we included 34 patients (mean age $74.6 \pm 8.6$ years), who underwent a SDCT of the chest and had a CIED in place. CI, MAR, VMI, and VMI $\mathrm{MAR}_{\text {(10 }}(10 \mathrm{keV}$ increment, range: 100-200 keV) were reconstructed. Mean and standard deviation of attenuation (HU) among hypo- and hyperdense artifacts adjacent to CIED generator and leads were determined using ROIs. Two radiologists qualitatively evaluated artifact reduction and diagnostic assessment of adjacent tissue. Results Compared to CI, MAR and $\mathrm{VMI}_{\mathrm{MAR}} \geq 100 \mathrm{keV}$ significantly increased attenuation in hypodense and significantly decreased attenuation in hyperdense artifacts at CIED generator and leads $(p<0.05)$. VMI $\geq 100 \mathrm{keV}$ alone only significantly decreased hyperdense artifacts at the generator $\left(p<0.05\right.$ ). Qualitatively, VMI $\geq 100 \mathrm{keV}, \mathrm{MAR}$, and $\mathrm{VMI}_{\mathrm{MAR}} \geq 100 \mathrm{keV}$ provided significant reduction of hyper- and hypodense artifacts resulting from the generator and improved diagnostic assessment of surrounding structures

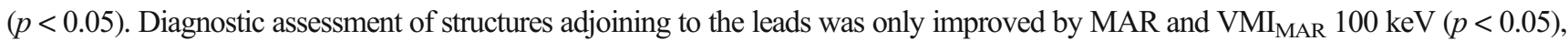
whereas $\mathrm{keV}$ values $\geq 140$ with and without MAR significantly worsened diagnostic assessment $(p<0.05)$.

Conclusions The combination of VMI and MAR as well as MAR as a standalone approach provides effective reduction of artifacts from CIEDs. Still, higher keV values should be applied with caution due to a loss of soft tissue and vessel contrast along the leads.

Key Points

- The combination of VMI and MAR as well as MAR as a standalone approach enables effective reduction of artifacts from CIEDs.

- Higher keV values of both VMI and VMI MAR at CIED leads should be applied with caution since diagnostic assessment can be hampered by a loss of soft tissue and vessel contrast.

- Recommended keV values for CIED generators are between 140 and $200 \mathrm{keV}$ and for leads around $100 \mathrm{keV}$.
\end{abstract}

Keywords Tomography, X-ray computed $\cdot$ Artifacts $\cdot$ Pacemaker

Lenhard Pennig

lenhard.pennig@uk-koeln.de

$1 \quad$ Institute for Diagnostic and Interventional Radiology, Faculty of Medicine and University Hospital Cologne, University of Cologne, Cologne, Germany

2 Department of Radiology, University Hospitals Cleveland Medical Center, Cleveland, OH, USA
3 Department of Radiology, Case Western Reserve University, Cleveland, OH, USA

4 Department of Radiology, Neuroradiology and Nuclear Medicine, Johannes Wesling University Hospital, Ruhr University Bochum, Bochum, Germany

5 Department of Radiology, Massachusetts General Hospital, Harvard Medical School, Boston, MA, USA

6 Else Kröner Forschungskolleg Clonal Evolution in Cancer, University Hospital Cologne, Cologne, Germany 


$\begin{array}{ll}\text { Abbreviations } \\ \text { CI } & \text { Conventional CT images } \\ \text { CIED } & \text { Cardiovascular implantable electronic devices } \\ \text { ICC } & \text { Intraclass correlation coefficient } \\ \text { ICD } & \text { Implanted cardioverter defibrillator } \\ \text { keV } & \text { Kiloelectron volt } \\ \text { MAR } & \text { Metal artifact reduction } \\ \text { MDCT } & \text { Multidetector computed tomography } \\ \text { ROI } & \text { Region of interest } \\ \text { SD } & \text { Standard deviation } \\ \text { SDCT } & \text { Spectral detector CT } \\ \text { VMI } & \text { Virtual monoenergetic images } \\ \text { VMI } & \text { Combination of MAR and VMI }\end{array}$

\section{Introduction}

Cardiovascular implantable electronic devices (CIEDs) such as permanent pacemakers, implantable cardioverter defibrillators, and cardiac resynchronization therapy devices improve outcome of various cardiac diseases and are increasingly used in aging populations [1,2]. Imaging of CIEDs is usually conducted after implantation and when complications - e.g., macrodislocation lead-dysfunctioning syndrome - are suspected. Conventional radiography represents the standard of care to evaluate physical integrity and positioning [2-4] with multidetector computed tomography (MDCT) of the chest being performed less frequently, e.g., for procedural planning of device lead extraction and assessment of lead perforation [5].

Metal artifacts arise as a combination of beam-hardening which results from absorption of low energetic photons [6,7], photon starvation which is caused by an insufficient amount of photons reaching the detector [7, 8], and scatter artifacts [9]. In CT scans, the metallic generator and leads of implanted CIEDs may cause hypo- and hyperdense artifacts, which impede assessment of adjacent structures. For instance, artifacts surrounding the pectoral CIED generator can hamper assessment of surrounding vessels, soft tissue, and lymph nodes [2] with the latter being especially of importance in oncologic patients, in which detection of lymph node or muscle metastases is relevant [10]. Likewise, pacemaker leads can cause strong artifacts peaking at the lead tip, which impair the assessment of vessel lumen and image interpretation of cardiac structures, such as chambers, valves, myocardium, and major thoracic vessels [2]. Consequently, the detection of lead-associated thrombosis, coronary/valve calcification, myocardial hypertrophy, and pericardial effusion can thereby be hampered [11, 12].

Previous studies have demonstrated the use of virtual monoenergetic images (VMI) from spectral detector CT (SDCT) and metal artifact reduction algorithms (MAR) for reduction of artifacts from implanted metal material as standalone techniques and as combined approaches $\left(\mathrm{VMI}_{\mathrm{MAR}}\right)$ [12-17].
To date, in SDCT imaging the combination of both methods has not been evaluated to reduce artifacts arising from CIEDs.

The objective of the study was to investigate the potential of VMI, MAR, and $\mathrm{VMI}_{\mathrm{MAR}}$ derived from venous phase SDCT of the chest to reduce artifacts surrounding CIED generator and leads. To this end, artifact reduction was objectively evaluated by examining the attenuation, or Hounsfield units (HU), respectively, of specific regions of interests (ROIs) around the CIED generator and leads. Additionally, artifact reduction was subjectively rated by two independent readers.

\section{Methods}

This retrospective study was approved by the local institutional review board (reference number 20-1068) and conducted in accordance with the ethical regulations of the 1964 Helsinki declaration including later amendments. The local institutional review board waived the necessity for informed consent.

\section{Patient population}

Patient scans were retrospectively selected from our internal database including data between March and December 2019 applying the following inclusion criteria:

(i) Contrast-enhanced, venous phase SDCT examinations of the thorax

(ii) Presence of a CIED with pectoral placement of the CIED generator and at least one lead with the respective tip positioned in a heart chamber

(iii) Availability of MAR reconstructions in addition to conventional and spectral image reconstructions

(iv) Age: $\geq 18$ years

There were no explicit exclusion criteria.

\section{Imaging protocol}

All patients were scanned head-first supine on a clinical SDCT (IQon, Philips Healthcare). No scans were explicitly performed for the purpose of this study. Iodinated contrast media (Accupaque $350 \mathrm{mg} / \mathrm{ml}$, GE Healthcare) was administered into an antecubital vein with scans being initialized with a 20- (thorax) or 50-s delay (thorax and abdomen) after exceeding a threshold value of $150 \mathrm{HU}$ in the descending aorta. The following scan parameters were used: matrix $512 \times 512$, collimation $64 \times 0.625 \mathrm{~mm}$, rotation time (thorax) $0.4 \mathrm{~s} /$ (thorax and abdomen) $0.33 \mathrm{~s}$, pitch (thorax) $1.015 /$ (thorax and abdomen) 0.671 , tube voltage $120 \mathrm{kVp}$. All examinations were conducted using automatic tube current modulation (DoseRight 3D-DOM, Philips Healthcare). Dose right index 
for the thorax examinations was 13 and for the thoracoabdominal examinations 17.

\section{Image post-processing}

Image reconstructions were performed using a hybriditerative reconstruction algorithm with a standard soft tissue kernel using the following specifications:

1. Conventional CT images (iDose4, level 3, filter B, Philips Healthcare; referred to as $\mathrm{CI}$ )

2. MAR (O-MAR, filter B, Philips Healthcare)

3. VMI (spectral B, level 3, Philips Healthcare; range: 100200 kiloelectron volt $(\mathrm{keV})$, increment of $10 \mathrm{keV})$

4. Combination of MAR and VMI (spectral B, level 3, range: $100-200 \mathrm{keV}$, increment of $10 \mathrm{keV}$; referred to as $\mathrm{VMI}_{\mathrm{MAR}}$ ).

For all reconstructions, slice thickness was set to $2 \mathrm{~mm}$ with an overlap of 50\%. MAR reconstructions were only performed on demand when artifacts from CIED were impairing diagnostic assessment. On the contrary, VMI reconstructions can be derived from SDCT after every examination.

\section{Objective analysis}

Image assessment was performed by a radiologist with 3 years of experience in chest imaging using a ROI-based method with a consistent size of approximately $50 \mathrm{~mm}^{2}$. ROIs were placed in CI and copied to MAR, VMI, and VMI $\mathrm{VAR}_{\text {MAsing }}$ the vendor's proprietary image viewer (IntelliSpace Portal, v9; Philips Healthcare).

ROIs were located in pronounced hypo- and hyperdense artifacts surrounding the CIED generator and the tip of CIED leads. Furthermore, for each ROI in artifact-impaired tissue, a $\mathrm{ROI}$ in the corresponding artifact-free reference tissue was also placed. For instance, when a hyperdense artifact impaired the ventricle lumen, an artifact-free region within the ventricle lumen was selected as the reference tissue. For each patient, seven ROIs were placed each in CI and MAR (reference tissue for artifact-free ventricle lumen could be used for the hypoand hyperdense artifact around the tip of CIED leads) and then copied to the VMI at the reconstructed keV levels. The mean and standard deviation (SD) of attenuation within each ROI was recorded. We considered the SD in artifact-impaired tissue indicative for artifact burden [18], although it needs to be considered that SD depends on several factors that might bias artifact reduction and although changes in SD can be rather subtle even though changes in mean attenuation can be large.

The corrected attenuation for hypo- and hyperdense artifacts was calculated as the difference of attenuation in artifactimpaired and artifact-free reference tissue [13]. This method considers general changes in attenuation along altering $\mathrm{keV}$ values of VMI to minimize potential bias to only detect real artifacts and artifact reduction [19]. Furthermore, corrected image noise [20] was calculated as the difference between image noise in artifact-impaired and artifact-free reference tissue. This method accounts for the general lower image noise in VMI with higher keV [19].

\section{Subjective analysis}

Two radiologists with 2 and 3 years of experience in chest imaging independently evaluated images on the same Picture Archiving and Communication System workstation (IMPAX EE release 20; Agfa HealthCare N.V.). Readers were blinded to clinical and patient data as well as to the results of the objective analysis. Full blinding towards reconstructions appeared not feasible for a consistent evaluation because of their distinct visual characteristics. Therefore, in each patient, readers were given a complete set of images, including CI, VMI, and $\mathrm{VMI}_{\mathrm{MAR}}$. $\mathrm{KeV}$ values for VMI and $\mathrm{VMI}_{\mathrm{MAR}}$ were 100,140 , and $200 \mathrm{keV}$. The larger increments compared to the objective analysis were chosen to allow for detection of relevant changes in image assessment. These might otherwise be obscured when images are rated that are too similar due to smaller $\mathrm{keV}$ value increment. Image parameters were as follows: axial plane, initial window level: 60 , and window width: 360 . Readers were allowed to adjust the window settings manually.

Readers were instructed to assess the extent of hypo- and hyperdense artifacts surrounding CIED generator and leads using a 5-point Likert scale (Table 1). Alike, they evaluated the diagnostic assessment of tissue adjacent CIED generator as well as cardiac structures and major vessels surrounding the CIED leads on a 5-point Likert scale (Table 1); readers considered artifact reduction capabilities but also a loss of soft tissue/vessel contrast that can appear at higher $\mathrm{keV}$ values in VMI and new artifacts that can be introduced by application of VMI as well as MAR [13, 21, 22].

\section{Statistical analysis}

Statistical analysis was performed with JMP software (release 14.1.0, SAS Institute). Quantitative results are shown as mean $\pm \mathrm{SD}$ with qualitative results being displayed as median with 10/90 percentile. The Shapiro-Wilk test was used to test for normal distribution. The Wilcoxon test with Steel adjustment for multiple comparisons was performed to test for significant differences. The statistical significance value was defined as $p<0.05$. Interreader agreement was assessed by calculating the intraclass correlation coefficient (ICC). Agreement was considered excellent when ICC $>0.74$, good when ICC $=0.60-0.74$, fair when ICC $=$ $0.40-0.59$, and poor when ICC $<0.4$ [23]. 
Table 1 Likert scale for subjective analysis of artifact reduction. CIED, cardiac implantable electric device; $C I$, conventional images; MAR, metal artifact reduction algorithm

\begin{tabular}{|c|c|}
\hline \multirow{5}{*}{$\begin{array}{l}\text { Extent of hypo- and hyperdense artifacts surrounding } \\
\text { CIED generator and leads }\end{array}$} & (5) Artifacts are absent/almost absent \\
\hline & (4) Minor artifacts \\
\hline & (3) Moderate artifacts \\
\hline & (2) Pronounced artifacts \\
\hline & (1) Massive artifacts \\
\hline \multirow{5}{*}{$\begin{array}{l}\text { Diagnostic assessment of pectoral soft } \\
\text { tissue surrounding CIED generator, } \\
\text { e.g., lymph nodes, muscles, } \\
\text { fat, and vessels }\end{array}$} & $\begin{array}{l}\text { (5) Fully diagnostic quality by no } \\
\text { artifacts/almost no artifacts }\end{array}$ \\
\hline & $\begin{array}{l}\text { (4) Marginally affected diagnostic } \\
\text { interpretability by minor streaks }\end{array}$ \\
\hline & $\begin{array}{l}\text { (3) Hampered diagnostic interpretability by } \\
\text { moderate artifacts }\end{array}$ \\
\hline & $\begin{array}{l}\text { (2) Restricted diagnostic interpretability by } \\
\text { strong artifacts }\end{array}$ \\
\hline & (1) Insufficient diagnostic interpretability \\
\hline \multirow{5}{*}{$\begin{array}{l}\text { Diagnostic assessment of the heart and major } \\
\text { associated vessels adjacent to CIED leads } \\
\text { regarding heart chambers, myocardium, } \\
\text { and pericardium, also considering potential } \\
\text { cardiac pathologies, e.g., thrombosis, calcification, } \\
\text { myocardial hypertrophy, and pericardial effusion }\end{array}$} & $\begin{array}{l}\text { (5) Full diagnostic quality/certainty without } \\
\text { artifacts/almost no artifacts }\end{array}$ \\
\hline & $\begin{array}{l}\text { (4) Marginally affected diagnostic } \\
\text { quality/certainty by minor streaks }\end{array}$ \\
\hline & $\begin{array}{l}\text { (3) Hampered diagnostic quality/certainty } \\
\text { by moderate artifacts }\end{array}$ \\
\hline & $\begin{array}{l}\text { (2) Restricted diagnostic quality/certainty by } \\
\text { strong artifacts }\end{array}$ \\
\hline & (1) Insufficient diagnostic quality/certainty \\
\hline
\end{tabular}

\section{Results}

\section{Study population and baseline characteristics}

Thirty-four patients were included in this study (mean age $74.6 \pm$ 8.6 years, 11 females). Seven patients had a single-chamber implanted cardioverter defibrillator (ICD), 26 patients a dualchamber ICD, and one patient had a cardiac resynchronization therapy device placed. The manufacturers and models of the CIED generator and leads are provided in Table 1 of the supplementary material. CT scans of the thorax were performed in one patient, and of the abdomen and thorax in 33 patients.

\section{Objective analysis}

\section{CIED generator}

Results of objective analysis of artifact reduction at CIED generator are provided in Table 2 and Fig. 1. Compared to $\mathrm{CI}$, in higher $\mathrm{keV}$ of $\mathrm{VMI}$, the corrected attenuation within hypodense artifacts was increased (e.g., CI/VMI $200 \mathrm{keV}$ :$78.9 \pm 129.5 / 4.3 \pm 119.6 \mathrm{HU}, p>0.05)$ and decreased within hyperdense artifacts (e.g., CI/VMI $200 \mathrm{keV}$ : $171.4 \pm 165.4 / 32.3$ $\pm 189.8 \mathrm{HU}, p<0.05$ ), for the latter with statistical significant differences at $\geq 100 \mathrm{keV}$. MAR and $\mathrm{VMI}_{\mathrm{MAR}} \geq 100 \mathrm{keV}$ significantly increased/decreased corrected attenuation in hypo-/hyperdense artifacts $(p<0.05)$.

VMI provided decreased corrected image noise in hypo- and hyperdense artifacts at all $\mathrm{keV}$ values, albeit this effect was not statistically significant $(p>0.05)$. However, MAR $(p<0.05)$ and $\mathrm{VMI}_{\mathrm{MAR}} \geq 100 \mathrm{keV}(p<0.05)$ enabled a significant reduction of corrected image noise in hypo- and hyperdense artifacts.

\section{CIED leads}

Results of objective analysis of artifact reduction around CIED leads are given in Table 3 and Fig. 2. Compared to $\mathrm{CI}$ in $\mathrm{VMI} \geq 100 \mathrm{keV}$, corrected attenuation in hypo- and hyperdense artifacts was comparable without yielding statistical significance $(p>0.05)$. MAR alone provided a significant increase of attenuation in hypodense $(\mathrm{CI} /$ MAR: $-127.5 \pm 77.3 /-59.7 \pm 50.4 \mathrm{HU} ; p<0.05)$ and reduction in hyperdense (CI/MAR: $51.8 \pm 37.7 / 22.3 \pm$ 30.5; $p<0.05$ ) artifacts. Likewise, $\mathrm{VMI}_{\mathrm{MAR}} \geq 100 \mathrm{keV}$ yielded a significant increase and decrease in corrected attenuation for hypo- and hyperdense artifacts, respectively $(p<0.05)$.

Corrected image noise in CI and VMI $\geq 100 \mathrm{keV}$ was comparable in hypo- and hyperdense artifacts $(p<0.05)$. However, MAR and $\mathrm{VMI}_{\mathrm{MAR}} \geq 100 \mathrm{keV}$ provided a significant decrease of image noise in both hypo- and hyperdense artifacts.

\section{Subjective analysis}

\section{CIED generator}

Results of subjective analysis of artifact reduction and surrounding tissue at CIED generator are given in Table 4. 
Table 2 Objective analysis of artifact reduction and surrounding tissues at CIED generator. Data is reported as mean $\pm \mathrm{SD}$. $C I$, conventional images; $V M I$, virtual monoenergetic images; $M A R$, metal artifact reduction algorithm; $V M I_{M A R}$, combination of MAR and VMI. Bold indicates significant changes in $\mathrm{HU}$ values compared to CI

\begin{tabular}{|c|c|c|c|c|}
\hline & \multicolumn{2}{|c|}{ Corrected attenuation } & \multicolumn{2}{|c|}{ Corrected image noise } \\
\hline & $\begin{array}{l}\text { Hypodense } \\
\text { artifacts }\end{array}$ & $\begin{array}{l}\text { Hyperdense } \\
\text { artifacts }\end{array}$ & $\begin{array}{l}\text { Hypodense } \\
\text { artifacts }\end{array}$ & $\begin{array}{l}\text { Hyperdense } \\
\text { artifacts }\end{array}$ \\
\hline CI & $-78.9 \pm 129.5$ & $171.4 \pm 165.4$ & $69.9 \pm 54.9$ & $55.2 \pm 92.0$ \\
\hline \multicolumn{5}{|l|}{ VMI } \\
\hline $100 \mathrm{keV}$ & $-37.9 \pm 97.8$ & $96.6 \pm 145.6$ & $56.9 \pm 62.2$ & $50.7 \pm 57.8$ \\
\hline $140 \mathrm{keV}$ & $-8.8 \pm 110.2$ & $52.3 \pm 173.5$ & $50.6 \pm 64.9$ & $46.4 \pm 53.8$ \\
\hline $200 \mathrm{keV}$ & $4.3 \pm 119.6$ & $32.3 \pm 189.8$ & $49.4 \pm 66.3$ & $46.3 \pm 52.5$ \\
\hline MAR & $18.4 \pm 69.5$ & $70.1 \pm 50.0$ & $13.3 \pm 13.2$ & $17.0 \pm 13.2$ \\
\hline \multicolumn{5}{|l|}{$\mathrm{VMI}_{\mathrm{MAR}}$} \\
\hline $100 \mathrm{keV}$ & $10.2 \pm 46.2$ & $28.3 \pm 50.0$ & $8.5 \pm 9.0$ & $13.0 \pm 11.2$ \\
\hline $140 \mathrm{keV}$ & $10.1 \pm 40.7$ & $10.5 \pm 56.2$ & $6.5 \pm 8.4$ & $10.8 \pm 10.8$ \\
\hline $200 \mathrm{keV}$ & $10.0 \pm 39.8$ & $2.5 \pm 59.7$ & $5.8 \pm 8.3$ & $10.1 \pm 10.7$ \\
\hline \multicolumn{5}{|l|}{$p$ value } \\
\hline CI vs. VMI 100-200 keV & $>0.05$ & $<0.05$ & $>0.05$ & $>0.05$ \\
\hline CI vs. MAR & $<0.05$ & $<0.05$ & $<0.05$ & $<0.05$ \\
\hline $\begin{array}{l}\text { CI vs. VMI } \\
\text { keV }\end{array}$ & $<0.05$ & $<0.05$ & $<0.05$ & $<0.05$ \\
\hline
\end{tabular}

$\mathrm{VMI} \geq 100 \mathrm{keV}, \mathrm{MAR}$, and $\mathrm{VMI}_{\mathrm{MAR}}$ provided significant reduction of hypo- and hyperdense artifacts compared to CI $(p<0.05)$. Diagnostic assessment of tissue adjacent to CIED generator significantly improved in VMI $\geq 100 \mathrm{keV}$, MAR, and $\mathrm{VMI}_{\mathrm{MAR}}$ at $140 \mathrm{keV}$ and $200 \mathrm{keV}$.

\section{CIED leads}

Table 5 displays the results of subjective analysis of artifact reduction and surrounding tissue at CIED leads. Only MAR and $\mathrm{VMI}_{\mathrm{MAR}} \geq 100 \mathrm{keV}$ provided significant reduction of hypodense
Fig. 1 Box-plot diagram displaying corrected attenuation values within hypo- and hyperdense artifacts adjacent to CIED generator in conventional CT images (conventional), virtual monoenergetic images (VMI, 100-200 keV), metal artifact reduction (MAR) algorithms, and their combination. HU, Hounsfield units

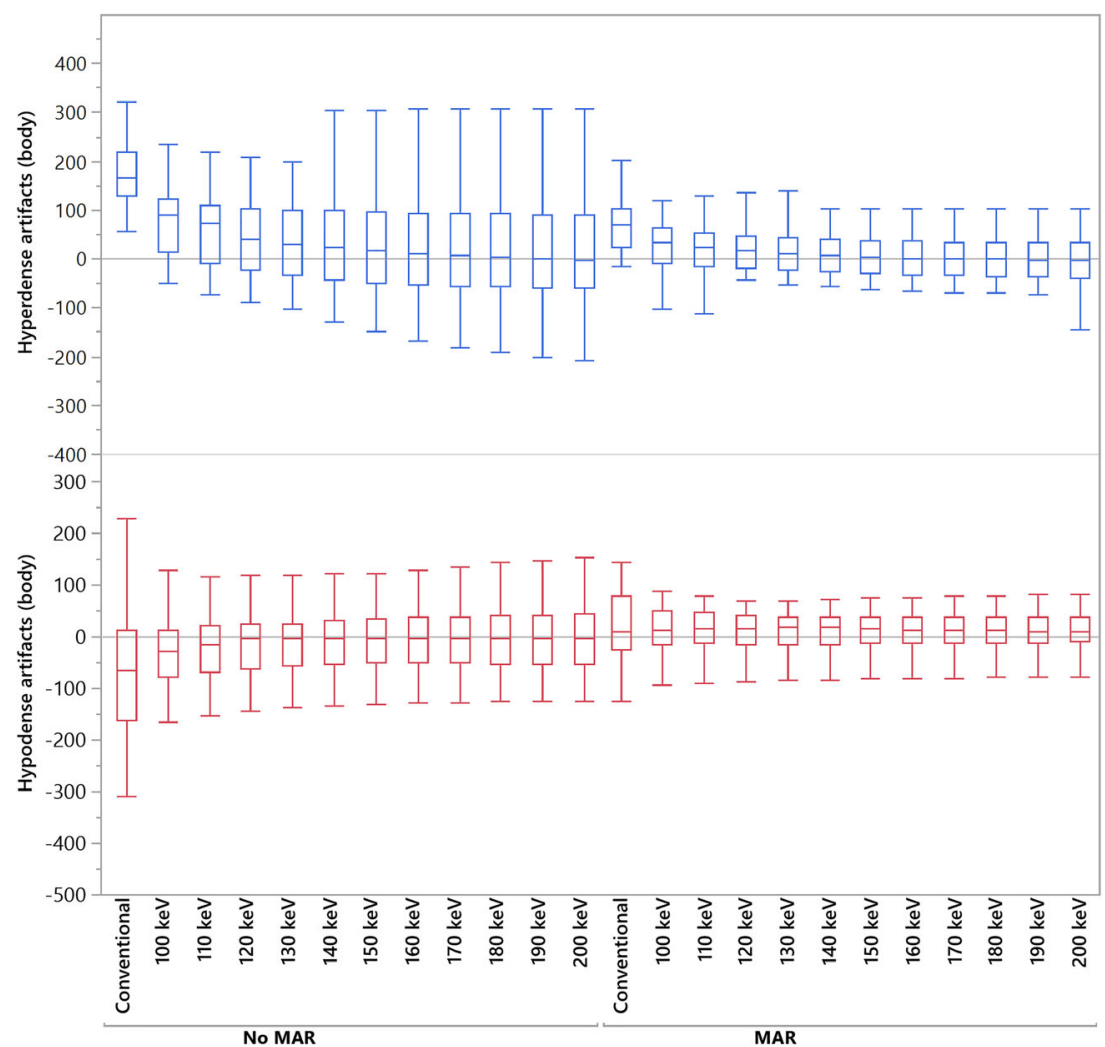


Table 3 Objective analysis of artifact reduction and surrounding tissues at CIED leads. Data is reported as mean $\pm \mathrm{SD}$. $C I$, conventional images; $V M I$, virtual monoenergetic images; MAR, metal artifact reduction algorithm; $V M I_{M A R}$, combination of MAR and VMI. Bold indicates significant changes in $\mathrm{HU}$ values compared to $\mathrm{CI}$

\begin{tabular}{|c|c|c|c|c|}
\hline & \multicolumn{2}{|c|}{ Corrected attenuation } & \multicolumn{2}{|c|}{ Corrected image noise } \\
\hline & $\begin{array}{l}\text { Hypodense } \\
\text { artifacts }\end{array}$ & $\begin{array}{l}\text { Hyperdense } \\
\text { artifacts }\end{array}$ & $\begin{array}{l}\text { Hypodense } \\
\text { artifacts }\end{array}$ & $\begin{array}{l}\text { Hyperdense } \\
\text { artifacts }\end{array}$ \\
\hline $\mathrm{CI}$ & $-127.5 \pm 77.3$ & $51.8 \pm 37.7$ & $66.5 \pm 46.1$ & $29.3 \pm 24.3$ \\
\hline \multicolumn{5}{|l|}{ VMI } \\
\hline $100 \mathrm{keV}$ & $-128.2 \pm 64.9$ & $47.6 \pm 32.9$ & $65.4 \pm 41.7$ & $30.1 \pm 24.4$ \\
\hline $140 \mathrm{keV}$ & $-127.2 \pm 62.6$ & $47.0 \pm 35.5$ & $64.9 \pm 41.8$ & $29.3 \pm 24.8$ \\
\hline $200 \mathrm{keV}$ & $-126.8 \pm 61.9$ & $46.7 \pm 37.0$ & $64.7 \pm 42.1$ & $29.1 \pm 25.1$ \\
\hline MAR & $-\mathbf{5 9 . 7} \pm \mathbf{5 0 . 4}$ & $22.3 \pm 30.5$ & $23.6 \pm 23.2$ & $9.3 \pm 12.9$ \\
\hline \multicolumn{5}{|l|}{$\mathrm{VMI}_{\mathrm{MAR}}$} \\
\hline $100 \mathrm{keV}$ & $-58.2 \pm 39.4$ & $19.7 \pm 24.6$ & $21.2 \pm 21.4$ & $10.6 \pm 10.7$ \\
\hline $140 \mathrm{keV}$ & $-\mathbf{5 7 . 1} \pm \mathbf{3 8 . 0}$ & $18.8 \pm 24.3$ & $20.4 \pm 22.1$ & $10.0 \pm 10.1$ \\
\hline $200 \mathrm{keV}$ & $-56.5 \pm 37.8$ & $18.4 \pm 24.7$ & $20.1 \pm 22.4$ & $9.7 \pm 9.9$ \\
\hline \multicolumn{5}{|l|}{$p$ value } \\
\hline CI vs. VMI 100-200 keV & $>0.05$ & $>0.05$ & $>0.05$ & $>0.05$ \\
\hline CI vs. MAR & $<0.05$ & $<0.05$ & $<0.05$ & $<0.05$ \\
\hline $\begin{array}{l}\text { CI vs. VMI } \\
\text { keV }\end{array}$ & $<0.05$ & $<0.05$ & $<0.05$ & $<0.05$ \\
\hline
\end{tabular}

artifacts $(p<0.05)$, whereas for hyperdense artifacts, all three techniques (VMI $\geq 100 \mathrm{keV}, \mathrm{MAR}$, and $\mathrm{VMI}_{\mathrm{MAR}}$ ) enabled significant decrease of artifacts $(p<0.05)$. Only MAR, VMI at 100 $\mathrm{keV}$, and $\mathrm{VMI}_{\mathrm{MAR}}$ at $100 \mathrm{keV}$ yielded a significant improvement for diagnostic assessment of tissue and cardiac structures adjacent to the leads. $\mathrm{KeV}$ values of 140 or higher in $\mathrm{VMI}$ and $\mathrm{VMI}_{\mathrm{MAR}}$ led to worsened diagnostic assessment.

Interreader agreement was good $(\mathrm{ICC}=0.66)$.

An illustrative case of artifact reduction around CIED generator and leads is presented in Fig. 3.
Fig. 2 Box-plot diagram displaying corrected attenuation values within hypo- and hyperdense artifacts adjacent to CIED leads in conventional CT images (conventional), virtual monoenergetic images (VMI, 100-200 keV), metal artifact reduction (MAR) algorithms, and their combination. HU, Hounsfield units

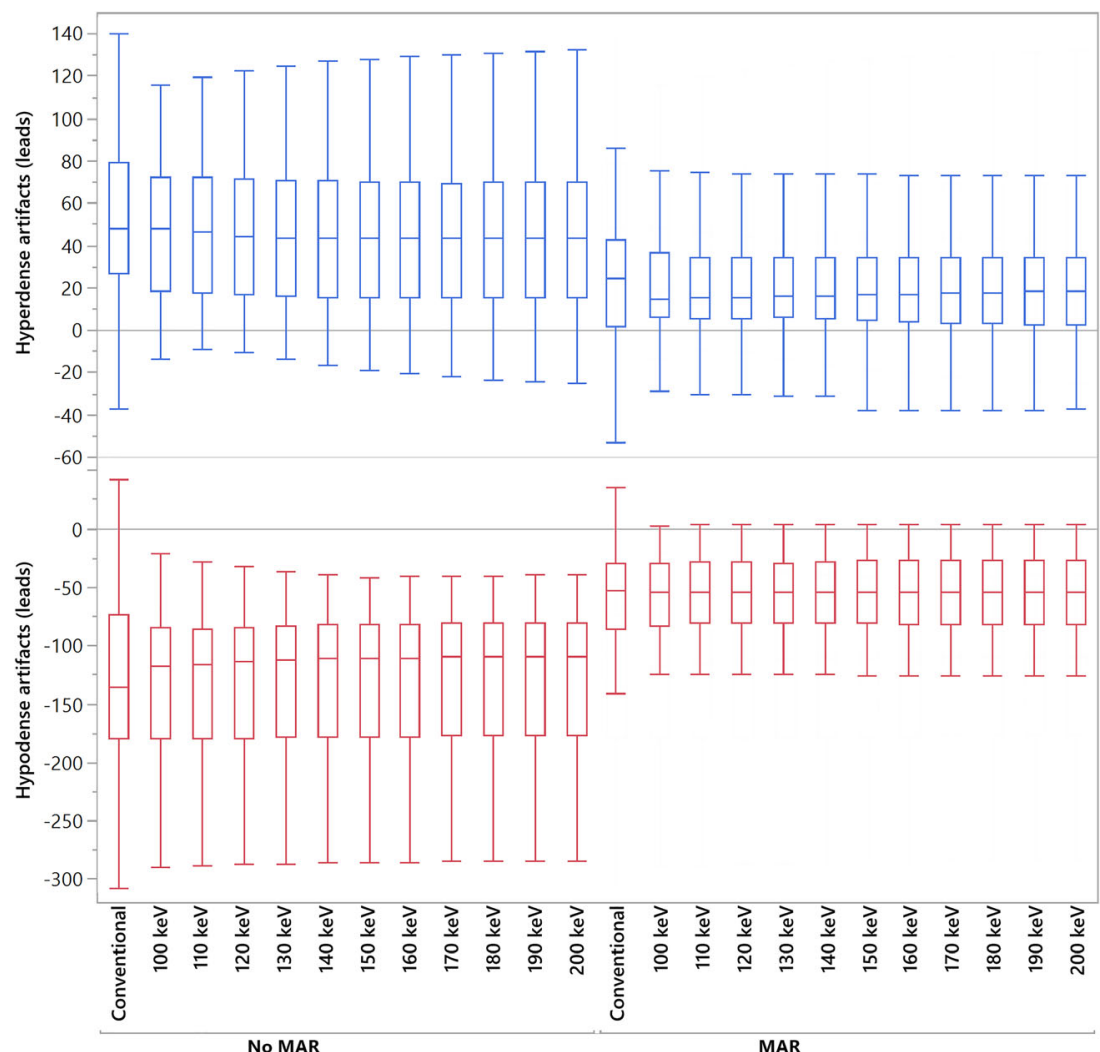


Table 4 Subjective analysis of artifact reduction and presence of new artifacts at CIED generator. Data is reported as median with $10 / 90$ percentile. $C I$, conventional images; VMI, virtual

monoenergetic images; MAR, metal artifact reduction algorithm; $V M I_{M A R}$, combination of MAR and VMI; ICC, intraclass correlation coefficient. Bold indicates significant changes in scores compared to $\mathrm{CI}$

\begin{tabular}{|c|c|c|c|}
\hline & \multicolumn{2}{|c|}{ Artifact extent } & \multirow{2}{*}{$\begin{array}{l}\text { Diagnostic assessmen } \\
\text { of surrounding tissue }\end{array}$} \\
\hline & Hypodense & Hyperdense & \\
\hline CI & $3(1-3)$ & $3(1-4)$ & $3(1-3)$ \\
\hline \multicolumn{4}{|l|}{ VMI } \\
\hline $100 \mathrm{keV}$ & $3(2-4)$ & $3(2-5)$ & $3.5(2-4)$ \\
\hline $140 \mathrm{keV}$ & $4(2-5)$ & $4(2-5)$ & $4(2-5)$ \\
\hline $200 \mathrm{keV}$ & $4(2-5)$ & $4(2-5)$ & $4(2-5)$ \\
\hline MAR & $3(2-4)$ & $4(3-4)$ & $3(3-4)$ \\
\hline \multicolumn{4}{|l|}{$\mathrm{VMI}_{\mathrm{MAR}}$} \\
\hline $100 \mathrm{keV}$ & $4(3-5)$ & $4(3-5)$ & $4(3-5)$ \\
\hline $140 \mathrm{keV}$ & $5(3-5)$ & $5(4-5)$ & $5(4-5)$ \\
\hline $200 \mathrm{keV}$ & $5(3-5)$ & $5(4-5)$ & $5(4-5)$ \\
\hline \multicolumn{4}{|l|}{$p$ value } \\
\hline CI vs. VMI $100 \mathrm{keV}$ & $<0.05$ & $<0.05$ & $<0.05$ \\
\hline CI vs. VMI $140 \mathrm{keV}$ & $<0.05$ & $<0.05$ & $<0.05$ \\
\hline CI vs. VMI $200 \mathrm{keV}$ & $<0.05$ & $<0.05$ & $<0.05$ \\
\hline CI vs. MAR & $<0.05$ & $<0.05$ & $<0.05$ \\
\hline CI vs. $\mathrm{VMI}_{\mathrm{MAR}} 100-200 \mathrm{keV}$ & $<0.05$ & $<0.05$ & $<0.05$ \\
\hline
\end{tabular}

\section{Discussion}

In this study, we assessed the performance of VMI, MAR, and their combination $\left(\mathrm{VMI}_{\mathrm{MAR}}\right)$ for reduction of artifacts from CIED generator and leads in SDCT imaging.

As major findings of this study, MAR and $\mathrm{VMI}_{\mathrm{MAR}}$ provided the most efficient artifact reduction in subjective and objective analysis while yielding improved diagnostic assessment of soft tissue and cardiac structures adjacent to CIED generator and leads. VMI at higher keV levels provided significant reduction of artifacts at CIED generator and leads (with exception of VMI for hypodense artifacts at the leads) in the subjective assessment, whereas a significant decrease of artifacts in the objective analysis was only observed for
Table 5 Subjective analysis of artifact reduction and presence of new artifacts at CIED leads. Data is reported as median with $10 / 90$ percentile. $C I$, conventional images; $V M I$, virtual monoenergetic images; $M A R$, metal artifact reduction algorithm; $V M I_{M A R}$, combination of MAR and VMI; $I C C$, intraclass correlation coefficient. Bold indicates significant changes in scores compared to $\mathrm{CI}$

\begin{tabular}{|c|c|c|c|}
\hline & \multicolumn{2}{|c|}{ Artifact extent } & \multirow{2}{*}{$\begin{array}{l}\text { Diagnostic assessment } \\
\text { of surrounding tissue }\end{array}$} \\
\hline & Hypodense & Hyperdense & \\
\hline CI & $3(3-4)$ & $2(2-3)$ & $2(2-3)$ \\
\hline \multicolumn{4}{|l|}{ VMI } \\
\hline $100 \mathrm{keV}$ & $3(3-4)$ & $3(2-4)$ & $3(2-3)$ \\
\hline $140 \mathrm{keV}$ & $3(3-4)$ & $3(2-4)$ & $2(1-4)$ \\
\hline $200 \mathrm{keV}$ & $3(3-4)$ & $3(3-4)$ & $1(1-4)$ \\
\hline MAR & $4(3-5)$ & $3(2-4)$ & $3(2-4)$ \\
\hline \multicolumn{4}{|l|}{$\mathrm{VMI}_{\mathrm{MAR}}$} \\
\hline $100 \mathrm{keV}$ & $4(3-5)$ & $4(3-4)$ & $3(2-4)$ \\
\hline $140 \mathrm{keV}$ & $4(3-5)$ & $4(3-5)$ & $2(1-4)$ \\
\hline $200 \mathrm{keV}$ & $4(3-5)$ & $4(3-5)$ & $1(1-4)$ \\
\hline \multicolumn{4}{|l|}{$p$ value } \\
\hline CI vs. VMI $100 \mathrm{keV}$ & $>0.05$ & $<0.05$ & $<0.05$ \\
\hline CI vs. VMI $140 \mathrm{keV}$ & $>0.05$ & $<0.05$ & $<0.05$ \\
\hline CI vs. VMI $200 \mathrm{keV}$ & $>0.05$ & $<0.05$ & $<0.05$ \\
\hline CI vs. MAR & $<0.05$ & $<0.05$ & $<0.05$ \\
\hline CI vs. VMI & $<0.05$ & $<0.05$ & $<0.05$ \\
\hline
\end{tabular}




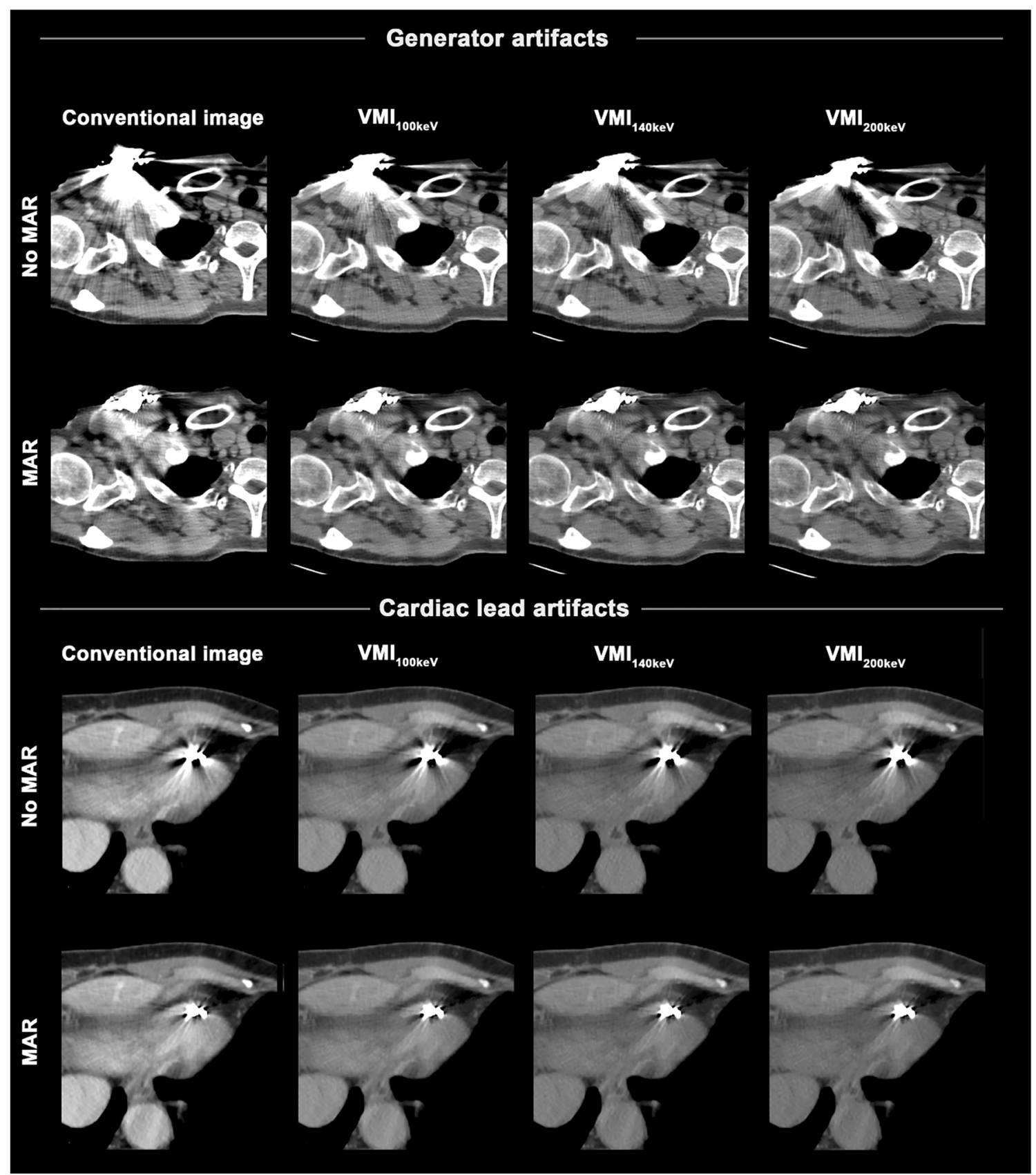

Fig. 3 Conventional images, virtual monoenergetic images (VMI, 100$200 \mathrm{keV}), \mathrm{MAR}$, and their combination $\left(\mathrm{VMI}_{\mathrm{MAR}}\right)$ in a 65 -year-old female patient with a dual-chamber implanted cardioverter defibrillator in place. For the CIED generator, VMI as a standalone (top row) approach allow for reduction of hyperdense and hypodense artifacts with best

hyperdense artifacts at CIED generator. Of note, VMI and $\mathrm{VMI}_{\mathrm{MAR}}$ led to relevant and significant decrease of diagnostic assessment at $\geq 140 \mathrm{keV}$ at CIED leads.

Prior studies have investigated the use of VMI, MAR, and their combination $\left(\mathrm{VMI}_{\mathrm{MAR}}\right)$ from dual-energy $\mathrm{CT}$ for reduction of artifacts arising from high density materials, such as orthopedic hardware [24], dental implants [13], deep brain stimulation leads [25], iodinated contrast agent [26], and coils performance at higher $\mathrm{keV}$ values, although relevant residual artifacts remain. These can be further reduced by MAR and $\mathrm{VMI}_{\mathrm{MAR}}$ (second row). For the CIED leads, VMI alone (third row) provide only minimal benefit in artifact reduction, whereas performance by $\mathrm{VMI}_{\mathrm{MAR}}$ (bottom row) is a lot stronger

and clips for intracranial aneurysm treatment [21, 27, 28]. More recent studies focusing on the reduction of artifacts impairing the assessment of the heart and surrounding structures (e.g., from pacemaker devices or other cardiac hardware) showed promising results for the application of artifact reduction algorithms and reconstruction techniques to improve image quality [12, 14-17]. For instance, Tatsugami et al. demonstrated that artifact reduction algorithms allow for a superior 
assessment of coronary arteries in patients with CIEDs [12]. Also, the application of a convolutional network has been successfully tested for the reduction of artifacts from pacemaker leads [16]. Van Hedent et al. investigated the application of VMI for the reduction of artifacts in chest and abdominal imaging of patients including artifacts from pacemakers [17].

Considering these previous studies, there has been uncertainty whether either method on its own (VMI or MAR) can provide sufficient artifact reduction. Particularly for stronger artifacts, e.g., those arising from CIED generator and leads, MAR and especially VMI as standalone approaches might yield suboptimal artifact reduction [13, 25, 29-31]. In line with these findings, our study demonstrated that, although VMI reduced artifacts in the visual assessment, it failed to significantly reduce artifacts in quantitative measurements, except for hyperdense artifacts at CIED generator. In contrast, MAR and $\mathrm{VMI}_{\mathrm{MAR}}$ enabled good artifact reduction for both kinds of artifacts and compartments of CIED as outlined in subjective and objective results. Still, this positive effect did not reflect on the diagnostic assessment of cardiac structures surrounding the CIED leads at higher $\mathrm{keV}$ values.

In line with previous studies, subjective artifact reduction at CIED generator and leads improved at higher $\mathrm{keV}$ levels in VMI and VMI $\mathrm{VAR}_{\text {I }}[13,32]$. However, this effect does not necessarily result in improved diagnostic assessment. This is due to the physical properties of VMI that accompanies higher $\mathrm{keV}$ values by the greater distance to the k-edge of iodine $(\sim 33 \mathrm{keV})$ [19], which at higher $\mathrm{keV}$ levels reduce contrast of soft tissue, vessel lumen, and cardiac chambers [19, 33]. This loss of contrast was found less severe around the CIED generator; therefore, diagnostic assessment was best at high $\mathrm{keV}$ values of $\geq 140$ $\mathrm{keV}$. Hence, we recommend $\mathrm{keV}$ values between 140 and $200 \mathrm{keV}$ to assess structures around the CIED generator. On the contrary, the loss of contrast of vessels and heart chambers significantly hampers the diagnostic assessment at higher $\mathrm{keV}$ values at the leads. Therefore, we recommend $100 \mathrm{keV}$ for optimal diagnostic assessment of structures adjacent to CIED leads. Nevertheless, as the optimal $\mathrm{keV}$ value differs between patients, these settings may need to be adjusted individually. A known limitation of VMI and MAR algorithms is that they can cause/produce new artifacts, which can impact the diagnostic quality of CT images [13, 21, 22]. Hence, we recommend the use of VMI and MAR only when artifacts are present, and CI should remain the standard of care. Of note, MAR was initially intended to be applied for orthopedic hardware. Still, as eluded above, several studies have shown its effectiveness also for non-orthopedic hardware $[13,21,25$, 34], e.g., deep brain stimulating electrodes [25]. However, in its white paper [22], the vendor does not recommend to apply MAR when metal is near air or low density tissue, e.g., in pacemakers, as the proximity to the lung can induce new artifacts [22]. Also, in smaller metal objects such as cardiac stents, the MAR algorithm might not alter image information as no or minimal metal is present $[22$, 25]. To this point, the vendor even disables MAR in dedicated cardiac examinations. Still, Große Hokamp et al. showed the dedicated value of MAR for reduction of artifacts from deep brain stimulating electrodes [25].

With the varying degrees of effectiveness for artifact reduction depending on implant type and its location within the human body, VMI and MAR algorithms for CT imaging are available from all major vendors $[19,35]$. However, their combined use is only possible in dual-energy CT. Nevertheless, single-energy CT scanners are by far much more common than DECT in clinical routine; thereby, MAR as a standalone post-processing approach has the advantage of a higher availability in conventional CT systems [24, 35]. Given the results of this study, MAR, not only in combination with VMI but also standalone, enables good reduction of artifacts arising from CIED generator and leads, which allows usage on conventional non-dual-energy CT systems.

\section{Limitations}

Besides its retrospective, single-center setting, our study has the following additional limitations that should be considered. First, the method to measure artifacts needs to be discussed. Due to its high feasibility, a relatively simple and standard approach in artifact reduction studies is ROI-based measurement of mean and standard deviation of attenuation $[13,36$, 37]. Nevertheless, more elaborate methods using dedicated artifact quantification algorithms might produce more precise results $[25,38]$. Second, given their differing physical properties, general changes in terms of attenuation and image noise appear in different keV levels of VMI [19]. To this end, we applied an intra-individual comparison between artifactimpaired tissue and correspondent unimpaired reference tissue resulting in corrected attenuation and corrected image noise, which enables the detection of real artifact reduction [13]. Third, full blinding of readers did not seem feasible since images (CI, VMI, and $\mathrm{VMI}_{\mathrm{MAR}}$ ) are distinguishable by their appearance. Furthermore, we aimed to encourage readers to also detect more subtle differences between reconstructions next to a qualitative rating of images; therefore, a full image set of one patient at a time was presented to the readers.

\section{Conclusions}

In presence of artifacts from CIED, the combination of VMI and MAR provides the most effective artifact reduction and improves diagnostic assessment of surrounding structures; however, MAR as a standalone approach also provides good 
artifact reduction. Still, higher $\mathrm{keV}$ values at CIED leads should be applied with caution due to a loss of soft tissue and vessel contrast, which impedes diagnostic accuracy. Based on our data, we recommend $\mathrm{keV}$ values between 140 and $200 \mathrm{keV}$ for artifacts surrounding the CIED generator and of $100 \mathrm{keV}$ for artifacts adjacent to the leads.

Supplementary Information The online version contains supplementary material available at https://doi.org/10.1007/s00330-021-07746-8.

Acknowledgements Clinician Scientist position supported by the Deans Office, Faculty of Medicine, University of Cologne.

Funding Open Access funding enabled and organized by Projekt DEAL.

\section{Declarations}

Guarantor The scientific guarantors of this publication are Lenhard Pennig and Kai Roman Laukamp.

Conflict of interest The authors of this manuscript declare relationships with the following companies: Philips Healthcare, Guerbet $\mathrm{GmbH}$

Lenhard Pennig: Study leave unrelated to this project as part of a research contract between Philips Healthcare and University Hospital Cologne.

David Zopfs: Received exemption from clinical duties for research outside this project as a part of a research agreement between Philips Healthcare and University Hospital Cologne.

Roman Gertz: Study leave unrelated to this project as part of a research contract between Philips Healthcare and University Hospital Cologne.

Nils Große Hokamp: Speaker's bureau, Philips Healthcare. Research support, Philips Healthcare.

Marcel Langenbach: Research grant, Guerbet GmbH.

Simon Lennartz: Research support, Philips Healthcare.

Jan Borggrefe: Speaker's bureau, Philips Healthcare.

Statistics and biometry No complex statistical methods were necessary for this paper.

Informed consent Written informed consent was waived by the Institutional Review Board.

Ethical approval Institutional Review Board approval was obtained.

Methodology
- retrospective
- observational
- performed at one institution

Open Access This article is licensed under a Creative Commons Attribution 4.0 International License, which permits use, sharing, adaptation, distribution and reproduction in any medium or format, as long as you give appropriate credit to the original author(s) and the source, provide a link to the Creative Commons licence, and indicate if changes were made. The images or other third party material in this article are included in the article's Creative Commons licence, unless indicated otherwise in a credit line to the material. If material is not included in the article's Creative Commons licence and your intended use is not permitted by statutory regulation or exceeds the permitted use, you will need to obtain permission directly from the copyright holder. To view a copy of this licence, visit http://creativecommons.org/licenses/by/4.0/.

\section{References}

1. Wong JA, Devereaux PJ (2019) Cardiac device implantation complications: a gap in the quality of care? Ann Intern Med 171:368369

2. Aguilera AL, Volokhina YV, Fisher KL (2011) Radiography of cardiac conduction devices: a comprehensive review. Radiographics 31:1669-1682

3. Aissa J, Boos J, Sawicki LM et al (2017) Iterative metal artefact reduction (MAR) in postsurgical chest CT: comparison of three iMAR-algorithms. Br J Radiol 90. https://doi.org/10.1259/bjr. 20160778

4. Lewis RK, Ehieli WL, Hegland DD et al (2020) Preprocedural computed tomography before cardiac implanted electronic device lead extraction: indication, technique, and approach to interpretation. J Cardiovasc Electrophysiol 31:723-732

5. Mak GS, Truong QA (2012) Cardiac CT: imaging of and through cardiac devices. Curr Cardiovasc Imaging Rep 5:328-336

6. Fayad LM, Patra A, Fishman EK (2009) Value of 3D CT in defining skeletal complications of orthopedic hardware in the postoperative patient. AJR Am J Roentgenol 193:1155-1163

7. Lee M-J, Kim S, Lee S-A et al (2007) Overcoming artifacts from metallic orthopedic implants at high-field-strength MR imaging and multi-detector CT. Radiographics 27:791-803

8. Mori I, Machida Y, Osanai M, Iinuma K (2013) Photon starvation artifacts of X-ray CT: their true cause and a solution. Radiol Phys Technol 6:130-141

9. Boas FE, Fleischmann D (2012) CT artifacts: causes and reduction techniques. Imaging Med 4:229-240

10. Lennartz S, Große Hokamp N, Abdullayev N et al (2019) Diagnostic value of spectral reconstructions in detecting incidental skeletal muscle metastases in CT staging examinations. Cancer Imaging 19:50

11. Kalisz K, Buethe J, Saboo SS, Abbara S, Halliburton S, Rajiah P (2016) Artifacts at cardiac CT: physics and solutions. Radiographics 36:2064-2083

12. Tatsugami F, Higaki T, Sakane H et al (2016) Coronary CT angiography in patients with implanted cardiac devices: initial experience with the metal artefact reduction technique. Br J Radiol 89: 20160493

13. Laukamp KR, Zopfs D, Lennartz S et al (2019) Metal artifacts in patients with large dental implants and bridges: combination of metal artifact reduction algorithms and virtual monoenergetic images provides an approach to handle even strongest artifacts. Eur Radiol 29:4228-4238

14. Aissa J, Thomas C, Sawicki LM et al (2017) Iterative metal artefact reduction in CT: can dedicated algorithms improve image quality after spinal instrumentation? Clin Radiol 72:428.e7-428.e12. https://doi.org/10.1016/j.crad.2016.12.006

15. Kikuchi N, Yanagawa M, Enchi Y et al (2020) The effect of the reconstruction algorithm for the pulmonary nodule detection under the metal artifact caused by a pacemaker. Medicine (Baltimore) 99 : e20579

16. Lossau (née Elss) T, Nickisch H, Wissel T, Morlock M, Grass M (2020) Learning metal artifact reduction in cardiac CT images with moving pacemakers. Med Image Anal 61. https://doi.org/10.1016/j. media.2020.101655

17. Van Hedent S, Kessner R, Große Hokamp N, Baran TZ, Kosmas C, Gupta A (2019) Metal artifact reduction in routine chest and abdominal examinations using virtual monoenergetic images from 
spectral detector computed tomography. J Comput Assist Tomogr 43:713-717

18. Große Hokamp N, Laukamp KR, Lennartz S et al (2018) Artifact reduction from dental implants using virtual monoenergetic reconstructions from novel spectral detector CT. Eur J Radiol 104:136142

19. McCollough CH, Leng S, Yu L, Fletcher JG (2015) Dual- and multi-energy CT: principles, technical approaches, and clinical applications. Radiology 276:637-653

20. Große Hokamp N, Neuhaus V, Abdullayev N et al (2017) Reduction of artifacts caused by orthopedic hardware in the spine in spectral detector CT examinations using virtual monoenergetic image reconstructions and metal-artifact-reduction algorithms. Skeletal Radiol 47:195-201

21. Zopfs D, Lennartz S, Pennig L et al (2020) Virtual monoenergetic images and post-processing algorithms effectively reduce CT artifacts from intracranial aneurysm treatment. Sci Rep 10. https://doi. org/10.1038/s41598-020-63574-8

22. (2012) Philips CT Clinical Science PHU. Metal Artifact Reduction for Orthopedic Implants (O-MAR). Accessed December 2020. Availabel at: http://clinical.netforum.healthcare.philips.com/us_en/ Explore/White-Papers/CT/Metal-Artifact-Reduction-for-O

23. Cicchetti DV (1994) Guidelines, criteria, and rules of thumb for evaluating normed and standardized assessment instruments in psychology. Psychol Assess 6:284-290

24. Wellenberg RHH, Hakvoort ET, Slump CH, Boomsma MF, Maas M, Streekstra GJ (2018) Metal artifact reduction techniques in musculoskeletal CT-imaging. Eur J Radiol 107:60-69

25. Große Hokamp N, Hellerbach A, Gierich A et al (2018) Reduction of artifacts caused by deep brain stimulating electrodes in cranial computed tomography imaging by means of virtual monoenergetic images, metal artifact reduction algorithms, and their combination. Invest Radiol 53:424-431

26. Laukamp KR, Gupta A, Große Hokamp N et al (2019) Role of spectral-detector $\mathrm{CT}$ in reduction of artifacts from contrast media in axillary and subclavian veins: single institution study in 50 patients. Acta Radiol 028418511986890

27. Pan YN, Chen G, Li AJ et al (2019) Reduction of metallic artifacts of the post-treatment intracranial aneurysms: effects of single energy metal artifact reduction algorithm. Clin Neuroradiol. https://doi. org/10.1007/s00062-017-0644-2

28. Fitsiori A, Martin SP, Juillet De Saint Lager A et al (2019) Iterative algorithms applied to treated intracranial aneurysms. Clin Neuroradiol 29:741-749
29. Dunet V, Bernasconi M, Hajdu SD, Meuli RA, Daniel RT, Zerlauth JB (2017) Impact of metal artifact reduction software on image quality of gemstone spectral imaging dual-energy cerebral CT angiography after intracranial aneurysm clipping. Neuroradiology 59: $845-852$

30. Pan Y-N, Chen G, Li A-J et al (2017) Reduction of metallic artifacts of the post-treatment intracranial aneurysms: effects of single energy metal artifact reduction algorithm. Clin Neuroradiol 1-8

31. Sunwoo L, Park S-W, Rhim JH et al (2018) Metal artifact reduction for orthopedic implants: brain CT angiography in patients with intracranial metallic implants. J Korean Med Sci 33:e158

32. Laukamp KR, Zopfs D, Wagner A et al (2019) CT artifacts from port systems: virtual monoenergetic reconstructions from spectraldetector $\mathrm{CT}$ reduce artifacts and improve depiction of surrounding tissue. Eur J Radiol 121:108733

33. Lennartz S, Laukamp KR, Neuhaus V et al (2018) Dual-layer detector CT of the head: initial experience in visualization of intracranial hemorrhage and hypodense brain lesions using virtual monoenergetic images. Eur J Radiol 108:177-183

34. Gupta A, Obmann VC, Jordan M et al (2021) CT artifacts after contrast media injection in chest imaging: evaluation of postprocessing algorithms, virtual monoenergetic images and their combination for artifact reduction. Quant Imaging Med Surg 11: 226-239

35. Huang JY, Kerns JR, Nute JL et al (2015) An evaluation of three commercially available metal artifact reduction methods for CT imaging. Phys Med Biol 60:1047-1067

36. Kidoh M, Nakaura T, Nakamura S et al (2014) Reduction of dental metallic artefacts in CT: value of a newly developed algorithm for metal artefact reduction (O-MAR). Clin Radiol 69:11-16

37. Bisschop R, Boomsma M, Van Raay JJA, Tiebosch ATMG, Maas M, Gerritsma CLE (2013) High prevalence of pseudotumors in patients with a Birmingham hip resurfacing prosthesis. J Bone Joint Surg Am 95:1554-1560

38. Mangold S, Gatidis S, Luz O et al (2014) Single-source dual-energy computed tomography: use of monoenergetic extrapolation for a reduction of metal artifacts. Invest Radiol 49:788-793

Publisher's note Springer Nature remains neutral with regard to jurisdictional claims in published maps and institutional affiliations. 